\title{
Experiences of staff members participating in primary care research activities: a qualitative study
}

This article was published in the following Dove Press journal:

International Journal of General Medicine

15 April 2015

Number of times this article has been viewed

\author{
Dominique Hange' \\ Cecilia Björkelund' \\ Irene Svenningsson ${ }^{2}$ \\ Marie Kivi ${ }^{3}$ \\ Maria C Eriksson' \\ Eva-Lisa Petersson ${ }^{1,2}$ \\ 'Department of Primary Health \\ Care/Public Health and Community \\ Medicine, Institute of Medicine, \\ Sahlgrenska Academy, University of \\ Gothenburg, Gothenburg, ${ }^{2 N a ̈ r h a ̈ l s a n ~}$ \\ Research and Development Primary \\ Health Care, Region Västra Götaland, \\ ${ }^{3}$ Department of Psychology, \\ University of Gothenburg, \\ Gothenburg, Sweden
}

Background: The aim of this paper was to study primary care staff members' experiences and perceptions of participating in a randomized controlled trial concerning Internet therapy.

Methods: Data were collected via five focus groups, each containing four to eight nurses or general practitioners. The systematic text condensation method described by Malterud was used for thematic analysis of meaning and content of data across cases.

Results: The informants believed it was important to conduct research within the primary care setting, but it was difficult to combine clinical work and research. They stressed also that there was a need for continuous information and communication between primary care centers and researchers as well as internally at each primary care center.

Conclusion: Staff members' experiences of participating in a research study were positive, although associated with various difficulties. It is important to include staff members when designing clinical studies; information should be given continuously during the study and communication facilitated between different occupational groups working at the primary care center.

Keywords: primary care, research, staff participation, Sweden

\section{Introduction}

Primary care is a sector with vast commitments and involving many patient visits. The infrastructure of primary care is complex, so research methods should be adapted to best fit this environment. Research is required to provide evidence-based knowledge, and scientific questions need to emanate from staff members who provide primary care services in order to develop evidence-based health care in this setting. ${ }^{1,2}$ A distinctive feature of primary care is that its activities are spread over large geographic areas, and encompass several different types of activity and multiple professions. An increasing amount of research activity is taking place in primary care centers (PCCs). ${ }^{3}$ However, there have been few Nordic studies concerning the experiences of staff members who participate in such research studies.

Recruiting participants for research studies requires careful planning, coordination, and flexibility. Close contact between researchers, health workers, and study personnel could result in successful patient recruitment. ${ }^{2,4}$ In a study in the north of England, around $10 \%$ of general practitioners (GPs) expressed an interest in research, although only $1 \%-2 \%$ were actually actively involved in research activity. ${ }^{1}$ Even if staff members have a positive attitude toward research, ${ }^{5}$ poor recruitment can be a problem. ${ }^{6,7}$ Some identified barriers to recruitment of patients into studies are: protection of the relationship between the health professional and the patient, lack of knowledge about
Correspondence: Dominique Hange Department of Primary Health Care/ Public Health and Community Medicine, Institute of Medicine, Sahlgrenska Academy, University of Gothenburg, PO Box 454, SE-405 30 Gothenburg, Sweden Tel +46317736843

Fax +46 3I 7781704

Email dominique.hange@vgregion.se submit your manuscript | www.dovepress.com

Dovepress

http://dx.doi.org//0.2147/IJGM.S78847
International Journal of General Medicine 2015:8 I43-148

(c) (i) (5) 2015 Hange et al. This work is published by Dove Medical Press Limited, and licensed under Creative Commons Attribution - Non Commercial (unported, v3.0) License. The full terms of the License are available at http://creativecommons.org/licenses/by-nc/3.0/. Non-commercial uses of the work are permitted without any further permission for the license are administered by Dove Medical Press Limited. Information on how to request permission may be found at: http://www.dovepress.com/permissions.php 
the study, and prioritization of clinical work rather than research. ${ }^{4,8,9}$

Starting in 2010, a randomized controlled intervention study (Primary Health Care-Internet [PRIM-NET]) concerning the effectiveness of Internet-mediated cognitive behavioral therapy (ICBT) in primary care was conducted in Västra Götaland, Sweden. However, the researchers had difficulty recruiting patients despite a prolonged enrolment period and increased efforts on the part of researchers and primary care staff members. The researchers were physically present at the PCCs during the PRIM-NET study, regularly emailed reminders and made telephone calls to the study nurses and psychologists, and held meetings with all staff members. Written information and a specific short reminder about the inclusion criteria were offered to each nurse and doctor for placement on their desks.

The authors, who had various durations of employment in PCCs, had both clinical experience and knowledge from the literature of the barriers when conducting research in these settings. This influenced the design of the ICBT study, but patient recruitment barriers still appeared during the study, indicating a need for a more in-depth understanding of factors influencing staff participation in research studies conducted in the PCC setting. ${ }^{10}$

The aim of this paper was to study primary care staff members' experiences and perceptions of participation in a randomized controlled study, as well as their views concerning scientific research activities in the workplace in general.

\section{Materials and methods}

All doctors and nurses $(n=31)$ at three of the 16 PCCs in Västra Götaland involved in the PRIM-NET ICBT study were invited to participate in the present study. One practice manager and one study nurse from each of the three clinics, ie, six individuals, were also invited to participate, resulting in 37 study participants in total. The study nurses worked at the PCCs but received $10 \%$ of their salary from PRIM-NET; therefore, they had a different role in the research and were thus interviewed individually. Three individuals declined to participate because of other engagements. Fifteen nurses, three practice managers, three study nurses, and 13 GPs participated, giving a total 34 individuals (eleven males and 23 females). The three PCCs that had participated in the PRIM-NET ICBT study for longest and had the most experience were asked to participate in the qualitative study. The randomized controlled study lasted 3 years, and it was not possible to wait for all PCCs to participate.
Five focus groups were conducted. ${ }^{11}$ Each group consisted of four to eight participants, comprising either solely nurses or solely doctors, based on the assumption that professional solidarity would deepen the discussion. The moderator (alternating between DH and ELP) initiated the discussion using an open-ended question inviting the participants to talk about their own experiences of research in PCCs. The managers and study nurses were interviewed separately, in order to reduce the potential influence of managers on staff members in the focus groups.

A focus group topic guide was developed that focused on the impact of the study on the staff members' daily work, their experiences of participating in the study, and the potential influence of the study on patients. A semistructured interview guide was developed for the managers and study nurses. ${ }^{12}$ Each meeting lasted no more than 1.5 hours and the interviews lasted about 1 hour. All sessions were audiotaped and transcribed verbatim. The data were collected in 2012 .

The study was approved by the regional ethical review board of Gothenburg. All participants gave their written informed consent to inclusion in the study, in accordance with the provisions of the Declaration of Helsinki.

\section{Analysis}

The analysis used systematic text condensation, inspired by Giorgi's phenomenological analysis according to Malterud. ${ }^{13,14}$ Systematic text condensation helps to develop new descriptions and concepts by presenting the participants' experiences, as expressed by them, rather than to explore the possible underlying meaning of what the informants said. The first step of the analysis was performed by DH and ELP, after which IS also collaborated in the analysis. With several researchers participating in the analysis, each contributed their perspective, but also strengthened each other's viewpoint. First, all material was read to obtain an overall impression and to categorize themes. Second, meaning units were identified that represented the respondents' experiences of working with research in PCCs. Third, the meaning units were condensed and labeled with codes and subcodes. Finally, the condensates were summarized to contents of each code group to a generalized description of the meaning of the text.

\section{Results}

Fifteen nurses, three managers, three study nurses, and 13 GPs participated in the study. Collection of data generated rich material for analysis. Participants shared their experiences of 
the difficulties they had concerning combining clinical work and research due to organizational demands. A major problem for the participants was finding and enrolling patients. Enrolment was complicated and time-consuming. The GPs experienced uncertainty with regard to whether or not ICBT was effective. These findings are elaborated on below and illustrated with quotations.

\section{Combining research and clinical work}

Most informants believed that it was important to conduct research within primary care, and with primary care patients. Informants expressed an interest in acquiring knowledge concerning research and being engaged in the research process. According to the participants, research was one of the areas that had less priority, and the current organization was not supporting research. Many GPs believed that their daily schedule was already fully booked, leaving no space for research activities, thus making it difficult to combine research and clinical work.

"It is important to participate in the study, but I will only participate in the study if there are recommendations from our boss".

The participants lacked continuous contact with the researchers during patient recruitment, which the participants believed resulted in fewer patients being included and decreased motivation to participate in the PRIM-NET study.

"It felt a bit like I was left out somehow. Before, researchers had come here regularly, now we lost that support. But we still had questions but so much to do so we did not take the time to call".

The workload at the PCC was high and there was not enough time for research activity. Several of the participants felt that they had no time to inform temporarily employed GPs about the study. Therefore, GPs with shorter terms of employment had less of an ability to understand the study process. The lack of time also affected the ability of permanent staff to collaborate.

"I can say that there was very little discussion about the study between the different professionals".

A contemporary national organizational change of primary care led to a reduction in staff members, which also reduced the enthusiasm for the study. The organizational change also involved competition between different units regarding reimbursement; this in turn negatively affected the study. Demands from management increased to produce "a certain way, a certain shape and a certain cost", as one GP put it. Economic compensation of the PCC for participating in the study had only a minimal impact.
"It is enough that we get some patients to remain on simvastatin instead of Lipitor, and we have earned this money equivalent to remuneration for participating in this study".

\section{Uncertainty concerning how to recruit patients}

"Patients with mental disorders are a common group in our PCC and should be easy to find". This was a frequently expressed view among the focus group participants concerning their ability to enroll patients to the PRIM-NET study. However, recruitment turned out to be more difficult than expected. Most nurses, and to a certain extent GPs, believed that the strict inclusion criteria were one of the main reasons for a lack of participation in the PRIM-NET study. Participants also perceived that patients who were randomly recruited to interventional Internet therapy were positive about their care, while those who received standard treatment were disappointed. This affected staff members negatively.

"Few patients qualified for the study and I think that also gives a resignation among those who recruit patients for the study. It affects nurses' motivation".

As one nurse described, "If GPs at the PCC had had a greater interest in mental illness, it would also have increased the competence among nurses". Many nurses expressed a lack of knowledge about mental illness. A telephone guidance nurse said that "the lack of knowledge regarding mental illness made me not go into deep discussions with the patient". Participants also reported that they lacked time to ask a sufficient number of questions about the patient's symptoms in the telephone conversation; this also reduced opportunities for inclusion.

The PCCs used different approaches to assess patients for study inclusion. Some had a model whereby the staff members referred patients to a specific psychosocial team which conducted the actual assessment. A study nurse was part of the team; thus, the nurses felt secure when it came to conducting the assessment. According to the informants, this probably led to a more stringent assessment, resulting in fewer recruited patients.

"We had the study in mind when we talked to the patients. But we have not taken a position on which patients should be included; it was made by the study nurse. So I do not think it has affected us in our work".

In other PCCs, the telephone guidance nurse made a rough assessment of the patient's suitability for the study before the study nurse conducted the final assessment. Because of a high workload, the telephone guidance nurse found it difficult to 
remember what to ask potential study participants about and this might have reduced the recruitment rate.

"If there had been more time to ask questions and more time to listen to the answers, we would surely have found more depressed patients".

The staff members had acquired new roles during participation in the study, which was perceived as constructive. One informant said that it actually took very little of her time because her role was to recruit patients to the PRIM-NET on the telephone and she "felt excited when patients agreed to participate". One informant believed that the project became her responsibility, and the GPs did not take the study seriously because she was a nurse. That a special time was set aside for the study was something that she thought was important to avoid, believing that others thought "that now I sit with the study again and do nothing".

\section{Reliable method or not}

The informants thought it essential to have both verbal and written information about the study presented to them in order to avoid having to read too much.

Since there were several GPs who left the PCC and other GPs who began to work at the PCC during the course of the study, information on the study did not reach everyone. Hence a lack of knowledge among informants (GPs) often arose regarding where current patients would be referred despite the information provided by the study nurse.

"When I started here, I got a little paper about the study. It was so short and I could not remember it".

The informants expressed a need for repeated information about the study from the researchers.

"The study has not had any direct imprint on either medical meetings or in our everyday work".

The informants also expressed a sense of insecurity regarding whether the ICBT method was reliable and effective. Not knowing exactly what happened to the patient during treatment felt like handing the patient over to the unknown. The informants also perceived a lack of access to the psychologist/psychotherapist. It was important for the informants to know who took responsibility for the patient after referral.

"I have no idea what I'm talking about when I say to the patient to go and sit in front of a computer. I have no idea and feel stupid not knowing".

According to the informants, staff members who were negative toward the ICBT method influenced the patient's attitude toward treatment and study participation. Informants emphasized the importance of remaining neutral to the method, in order to avoid transferring the staff members' opinion to the patient.

"I believe that some patients would rather talk to a human being than to a computer".

\section{Discussion}

Staff members' experiences of participating in the PRIM-NET study were positive although they reported various difficulties and uncertainties about the purpose of the study, the process of patient enrolment, and ICBT as a method. All informants emphasized the importance of participating in this PCC study. The main problem perceived by the informants was the difficulties of combining research and clinical work when each PCC was actively engaged in the study. In the primary care organization, little space for research activities was at hand. Moreover, recruiting patients was more complicated than the staff members expected. Staff members attributed recruitment difficulties to lack of knowledge about the study, reduced contact between study staff members and researchers due to time limitations and loss of organizational and management support. The informants felt dubious with regard to the effectiveness of ICBT, which affected both potential study patients and staff members.

\section{Validity and reflexivity}

Our sample of GPs and nurses presented their experiences openly, giving us the impression that we received honest answers, rather than answers being provided to meet our expectations. We chose to include only nurses or GPs in each focus group to create a safe atmosphere. This may have been a limitation because an interdisciplinary discussion of cooperation and communication between the various disciplines could have been narrowed.

Since PRIM-NET was ongoing for more than 2 years at the various PCCs, many changes regarding the circumstances of the GPs took place during that period. Our findings might be transferable to other PCCs in Sweden, since the prerequisites of many primary care centres in Sweden are similar. We conducted interviews or focus groups with almost all the GPs and nurses at three separate centers. All PCCs in this study were in the public sector. Individuals working at private PCCs might have given other answers.

All the authors have worked for several years in primary care settings. This could potentially influence both formulation of the topic guide and interpretation and analysis of the information. However, we believe that contextual knowledge is essential both when formulating research questions and interpreting the results, although this knowledge may have 
influenced our analysis of the material, albeit not to any significant extent.

\section{Staff members positive about scientific studies}

PRIM-NET might have been more successful if the staff members had been more involved from the outset; this would have increased their sense of ownership of the study. A way to succeed in performing a study in primary care is to have clinicians and researchers interested in designing studies that include elements of importance for effective and reality-adapted research. ${ }^{15}$

Health care staff members were in favor of research in PCC in principle, but actually being involved made matters more difficult than expected. Despite the fact that depressed patients were common at the PCCs, it was difficult to enroll them into the PRIM-NET study. Two different recruitment procedures were used, but this seemed not to make any difference concerning the rate of recruitment. When the recruitment process involved team assessment, the nurses applied strict inclusion criteria, which might have caused a reduced influx of patients, and recruitment involving assessment by a telephone guidance nurse was time-consuming, which could have been due to lack of practice but also to reduced influx. Problems associated with strict inclusion criteria are, however, a well-known phenomenon. Earlier studies have shown that in addition to strict inclusion criteria, lack of interest from GPs with regard to patients with depression and shortage of time are some difficulties that arise when enrolling patients into studies. ${ }^{16}$

The participants' uncertainty regarding how to recruit patients as well as lack of support from the study researchers indicate that more detailed feedback and communication as well as regular site visits concerning how to recruit patients are needed. ${ }^{17}$ Other reasons for not recruiting patients were forgetfulness and time pressure, which have also been reported in other studies. ${ }^{18}$ For the researcher, it is important to closely follow the study process at the individual PCC to be able to maintain good support as well as motivation to continue the study. ${ }^{19}$ Using established clinical networks in PCC may be another way to enhance recruitment of GPs. ${ }^{20}$

Some of the nurses alluded to a lack of a physician with a "special interest" in mental illness, and had such a person been available, they believed that this would improve the nurses' competence regarding depression. Lack of knowledge could also have been a reason for the low recruitment rate, ie, nurses might need more time to interview the potential patient for inclusion. ${ }^{21}$
We found some difficulties in communication between GPs and nurses with regard to who was responsible for the study and who had ownership of the study. The reason for this might be that the nurses did not feel confident in their new role as a study nurse, while at the same time having to acquire new knowledge and competence for their research roles. ${ }^{19}$ More regular feedback from the researchers during the study period might have facilitated the nurses' work with this. Collaboration between nurses and GPs has been explored mostly from the nurses' point of view in a hospital setting. ${ }^{22}$ However, to a lesser extent, the perceptions of GPs have been described as an area with opportunities for improvement. ${ }^{23}$

Some of the GPs expressed doubt about the treatment and a sense of "loss of control" over what happened to their patients when they were included in the study. This skepticism has been seen in other studies of Internet-based treatment. ${ }^{24}$ The GPs, although fascinated by the idea of computerized therapy, believed that the computer option was "too impersonal" and would not meet the needs of patients. ${ }^{24}$

\section{Conclusion}

When conducting a study within the primary care context, researchers should be physically present at the PCC during the major part of the study, supporting communication and feedback between staff members, the research team, and the individual staff members at the PCC. We emphasize the importance of scrutinizing the inclusion criteria in detail so that they are relevant to everyday practice and ensuring that PCCs and researchers are involved in the study as much as possible.

\section{Disclosure}

The authors report no conflicts of interest in this work.

\section{References}

1. Pearl A, Wright S, Gamble G, Doughty R, Sharpe N. Randomised trials in general practice - a New Zealand experience in recruitment. N Z Med J. 2003;116(1186):U681.

2. Askew DA, Clavarino AM, Glasziou PP, Del Mar CB. General practice research: attitudes and involvement of Queensland general practitioners. Med J Aust. 2002;177(2):74-77.

3. Murphy E, Spiegal N, Kinmonth AL. 'Will you help me with my research?' Gaining access to primary care settings and subjects. $\mathrm{Br} J$ Gen Pract. 1992;42(357):162-165.

4. Jones R. Primary care research: ends and means. Fam Pract. 2000;17(1): $1-4$.

5. Gaglio B, Nelson CC, King D. The role of rapport: lessons learned from conducting research in a primary care setting. Qual Health Res. 2006; 16(5):723-734.

6. Fletcher B, Gheorghe A, Moore D, Wilson S, Damery S. Improving the recruitment activity of clinicians in randomised controlled trials: a systematic review. BMJ Open. 2012;2(1):e000496. 
7. Sullivan-Bolyai S, Bova C, Deatrick JA, et al. Barriers and strategies for recruiting study participants in clinical settings. West J Nurs Res. 2007;29(4):486-500.

8. Mason V, Shaw A, Wiles N, et al. GPs' experiences of primary care mental health research: a qualitative study of the barriers to recruitment. Fam Pract. 2007;24(5):518-525.

9. Fallowfield L, Ratcliffe D, Souhami R. Clinicians' attitudes to clinical trials of cancer therapy. Eur J Cancer. 1997;33(13):2221-2229.

10. Foy R, Eccles M, Grimshaw J. Why does primary care need more implementation research? Fam Pract. 2001;18(4):353-355.

11. Morgan DL. Focus Groups as Qualitative Research. 2nd ed. Thousand Oaks, CA, USA: Sage Publications; 1997.

12. Kvale S. Interviews: An Introduction to Qualitative Research Interviewing. Thousand Oaks, CA, USA: Sage; 1996.

13. Malterud K. Shared understanding of the qualitative research process. Guidelines for the medical researcher. Fam Pract. 1993;10(2): 201-206.

14. Giorgi A. Phenomenology and Psychological Research. Pittsburgh, PA, USA: Duquesne University Press; 1985.

15. Roy-Byrne PP, Sherbourne CD, Craske MG, et al. Moving treatment research from clinical trials to the real world. Psychiatr Serv. 2003; 54(3):327-332.

16. Cranney M, Warren E, Barton S, Gardner K, Walley T. Why do GPs not implement evidence-based guidelines? A descriptive study. Fam Pract. 2001;18(4):359-363.
17. Rendell JM, Merritt RD, Geddes JR. Incentives and disincentives to participation by clinicians in randomised controlled trials. Cochrane Database Syst Rev. 2007;2:MR000021.

18. Gray RW, Woodward NJ, Carter YH. Barriers to the development of collaborative research in general practice: a qualitative study. $\mathrm{Br} \mathrm{J}$ Gen Pract. 2001;51(464):221-222.

19. Spilsbury K, Petherick E, Cullum N, Nelson A, Nixon J, Mason S. The role and potential contribution of clinical research nurses to clinical trials. J Clin Nurs. 2008;17(4):549-557.

20. Temte JL, Grasmick ME. Recruiting primary care clinicians for public health and bioterrorism surveillance. WMJ. 2009;108(2):104-108.

21. Sadler GR, Lantz JM, Fullerton JT, Dault Y. Nurses' unique roles in randomized clinical trials. J Prof Nurs. 1999;15(2):106-115.

22. Ajeigbe DO, McNeese-Smith D, Leach LS, Phillips LR. Nurse-physician teamwork in the emergency department: impact on perceptions of job environment, autonomy, and control over practice. J Nurs Adm. 2013; 43(3):142-148.

23. Onishi M, Komi K, Kanda K. Physicians'perceptions of physician-nurse collaboration in Japan: effects of collaborative experience. J Interprof Care. 2013;27(3):231-237.

24. Hetherton J, Matheson A, Robson M. Recruitment by GPs during consultations in a primary care randomized controlled trial comparing computerized psychological therapy with clinical psychology and routine GP care: problems and possible solutions. Prim Health Care Res Dev. 2006;5(01):5-10.
International Journal of General Medicine

\section{Publish your work in this journal}

The International Journal of General Medicine is an international, peer-reviewed open-access journal that focuses on general and internal medicine, pathogenesis, epidemiology, diagnosis, monitoring and treatment protocols. The journal is characterized by the rapid reporting of reviews, original research and clinical studies across all disease areas.

\section{Dovepress}

A key focus is the elucidation of disease processes and management protocols resulting in improved outcomes for the patient. The manuscript management system is completely online and includes a very quick and fair peer-review system. Visit http://www.dovepress.com/ testimonials.php to read real quotes from published authors. 